\title{
Software
}

\section{Los navegadores de la web 2.0: Firefox, Opera y Explorer}

\author{
Por Ricard Monistrol y Lluís Codina
}

Resumen: Web 2.0 y navegadores son dos conceptos estrechamente unidos. La razón es que los navegadores son los agentes de usuario que deben hacer realidad la web 2.0. Con una gran coincidencia en el tiempo han aparecido las nuevas versiones de los tres navegadores más importantes: Explorer, Firefox y Opera. En este artículo se examinan y se comparan nuevas capacidades y prestaciones de los tres navegadores mencionados frente a la realidad de la nueva web.

Palabras Clave: Agentes de usuario, Navegadores de internet, Explorer, Firefox, Opera, Web 2.0, Usabilidad, Accesibilidad.

\section{Title: Web browsers and Web 2.0}

Abstract: Web 2.0 and browsers are two very closely connected concepts. The reason is that browsers are the user agents that are needed to make navigating web 2.0 a reality. Within a very short span of time, new versions have been issued by the top three web browsers: Explorer, Firefox and Opera. In this article we examine and compare the new capabilities and functions of these three browsers in terms of the new web.

Keywords: User agents, Web browsers, Explorer, Firefox, Opera, Web 2.0, Usability, Accessibility.

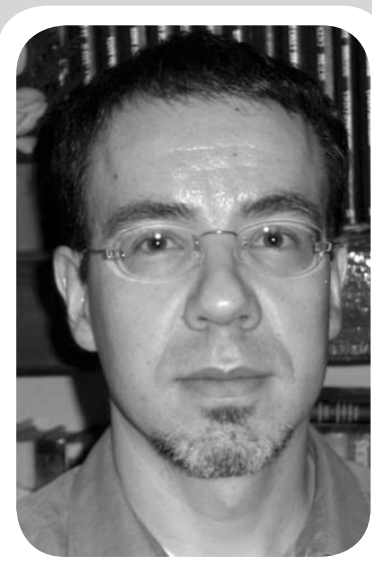

Ricard Monistrol, licenciado en periodismo por la Univ. Autónoma de Barcelona (UAB), diplomado en estudios avanzados en comunicación social $e$ investigador colaborador del grupo DigiDoc, IULA, UPF; es director de Culturcom, consultoría en comunicación y difusión cultural.

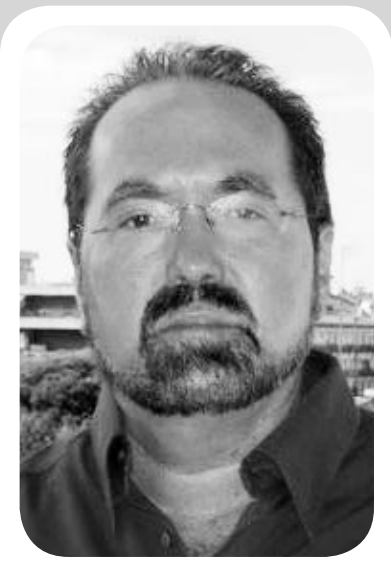

Lluís Codina es doctor en ciencias de la información y profesor titular de ciencias de la documentación de la Univ. Pompeu Fabra (UPF) de Barcelona. Coordina el grupo de investigación en documentación digital (DigiDoc) del Instituto Universitario de Lingüistica Aplicada (IULA) de la UPF.

Monistrol, Ricard; Codina, Lluís. "Los navegadores de la web 2.0: Firefox, Opera y Explorer”. En: El profesional de la información, 2007, mayo-junio, v. 16, n. 3, pp. 261-267.

DOI: 10.3145/epi.2007.may.11

\section{Introducción}

NO PARECE CASUALIDAD que Opera, Microsoft y Mozilla hayan lanzado sus nuevas versiones casi al unísono. ¿Los motivos? En el caso de Microsoft, sin duda un intento de volver a recuperar las posiciones perdidas en los últimos años. En el caso de Firefox y Opera consolidar y aumentar sus respectivas posiciones emergentes siempre a costa de Explorer.

En consecuencia, este año 2007 se perfila como el de la aparición de los navegadores "de segunda generación", a saber: Explorer 7, Firefox 2 y Opera 9. Lo curioso de toda esta situación no es que Explorer haya perdido cuota de mercado frente a los anteriores, sino que siga siendo el más utilizado a la vista de las prestaciones comparadas.

La importante es que el éxito de Firefox y la exquisita calidad de Opera han acabado con los años de plomo en los que Explorer parecía ser la única alternativa frente a un Netscape que no representaba una amenaza real para nadie dadas las absurdas e incompetentes versiones que sacaba al mercado en los últimos años.

Es de esperar que, dada la fuerte tendencia en favor de los estándares web, no vuelva a producirse una situación en la cual un navegador resulta tener la mayor cuota de mercado solamente porque los si- tios webs se hacen pensando en él. Crucemos los dedos.

Firefox es el segundo más usado a nivel global según las estadísticas, cifrándose su cuota de mercado global en alrededor de un $20 \%$. Aparecido en el año 2004, su gran aceptación mundial ha tenido un punto y aparte desde octubre de 2006, cuando presentó su versión actual: Firefox 2.0.

Por su parte, Opera, de origen noruego, es anterior a Firefox, ya que se remonta a 1994 y su origen empresarial ha determinado una evolución muy diferente. En los primeros años se vendió como aplicación de pago hasta que en diciembre de 2000 cambió radicalmente su 
modelo de negocio y se ofreció de forma gratuita, siempre que el usuario soportara publicidad en el navegador. Finalmente, desde 2005, con la versión 8.5, volvió a dar un giro y desde entonces se ofrece de forma completamente gratuita y sin necesidad de soportar publicidad.

\section{La web 2.0}

Si los orígenes de la web 2.0 son imprecisos, no sucede así con su denominación. Ésta última se sitúa claramente en el año 2004, cuando tuvo lugar su presentación "oficial" en el marco de una conferencia del mismo nombre en la ciudad de San Francisco (EUA). En aquella ocasión, Tim O'Reilly explicó que la idea se gestó en una reunión de tipo brainstorming con Dale Dougherty, vicepresidente de O'Reilly $\mathrm{Me}$ dia y Craig Line de Media Line.

Se supone que la www ha sido desde su inicio una plataforma en constante evolución y que la web 2.0 es la sublimación de esta idea mediante una serie de sitios que aportan servicios directos a los usuarios. Para los analistas tres son sus principales características:

- Las aplicaciones en línea, por ejemplo, la hoja de cálculo y el editor de textos de Google.

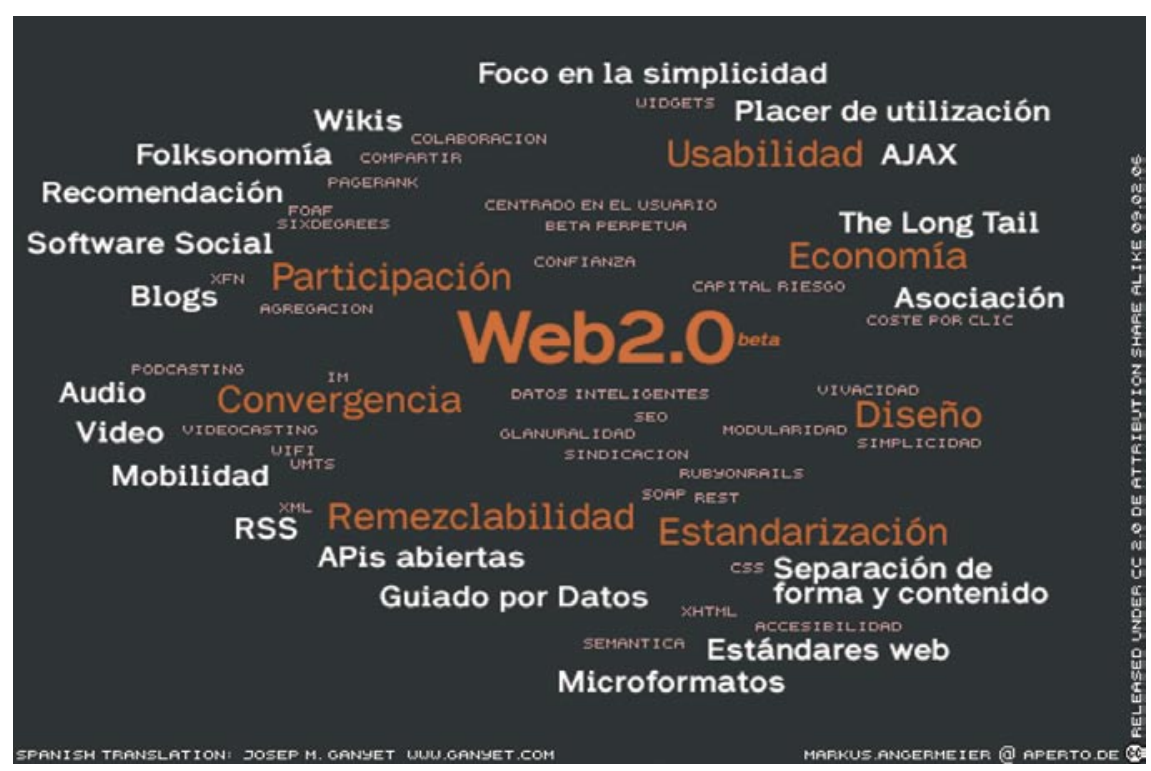

Figura 1. Póster sobre la web 2.0 de Markus Angermeier con ejemplos de aplicaciones y servicios (fuente: http://es.wikipedia.org/wiki/Imagen:Web2OmemeES.png)
- La participación, con casos como Flickr o Wikipedia.

- La sindicación de informaciones y servicios, como por ejemplo el uso de rss y de las API de Google.

Desde un punto de vista tecnológico, la web 2.0 se basa en AJAX (asynchronous javascript and $\mathrm{xml}$ ), lo que significa también que se basa en xhmtl y css ( $\mathrm{y}$ no en html con su lista de elementos depreciados o directamente no estándar). Con lo cual, en realidad la web 2.0 aporta un cuarto ingrediente que a veces pasa desapercibido: un nuevo y saludable respeto por la creación de sitios siguiendo estándares. Dicho de otro modo: los sitios de la web 2.0 no se pueden hacer pensado en un navegador concreto. Visto desde el otro lado, esto significa que los navegadores ahora tendrán cada vez una mayor capacidad para interpretar de forma correcta los estándares que marca el World Wide Web Consortium, así como deben incluir cada vez un mayor número de intérpretes: html, xml, xhtml, javascript, css, etc.

Existe también una parte oscura. Una nueva web asociada ahora al uso de herramientas implica también mayores riesgos. En primer

\section{"La web 2.0 aporta un ingrediente que a veces pasa inadvertido: respeto por la creación de sitios siguiendo estándares"}

lugar, en ocasiones conlleva facilitar datos personales, pero también ordenadores de usuarios más vulnerables porque deben aceptar más temporales en forma de controles Activex o de programas en Java por no mencionar las cookies. Por ello los nuevos navegadores deben cuidar ahora de forma especial no solamente su grado de cumplimiento de los estándares, sino también aspectos de seguridad.

En lo que sigue examinaremos las tres principales aplicaciones comentadas en relación con los aspectos de seguridad en la navegación. Además, tendremos en cuenta otros dos aspectos básicos de la web actual: la personalización y la accesibilidad.

\section{Seguridad}

Posiblemente es una de las prioridades de cualquier profesional o institución cuyos ordenadores trabajen en la Red. En este sentido resulta importante mantener actualizadas las protecciones activas tales como los antivirus y los buscadores de software malicioso/espía, sin descontar el sistema cortafuegos, que aisla el ordenador o la propia red institucional de posibles ataques de crackers.

Los nuevos navegadores ayudan también en estas cuestiones y aportan elementos de seguridad propios. Al menos, la información oficial en cualquiera de los tres casos estudiados indica que protege al usuario de sitios fraudulentos.

Tanto Explorer como Firefox activan de forma automática un filtro anti-phishing cada vez que 


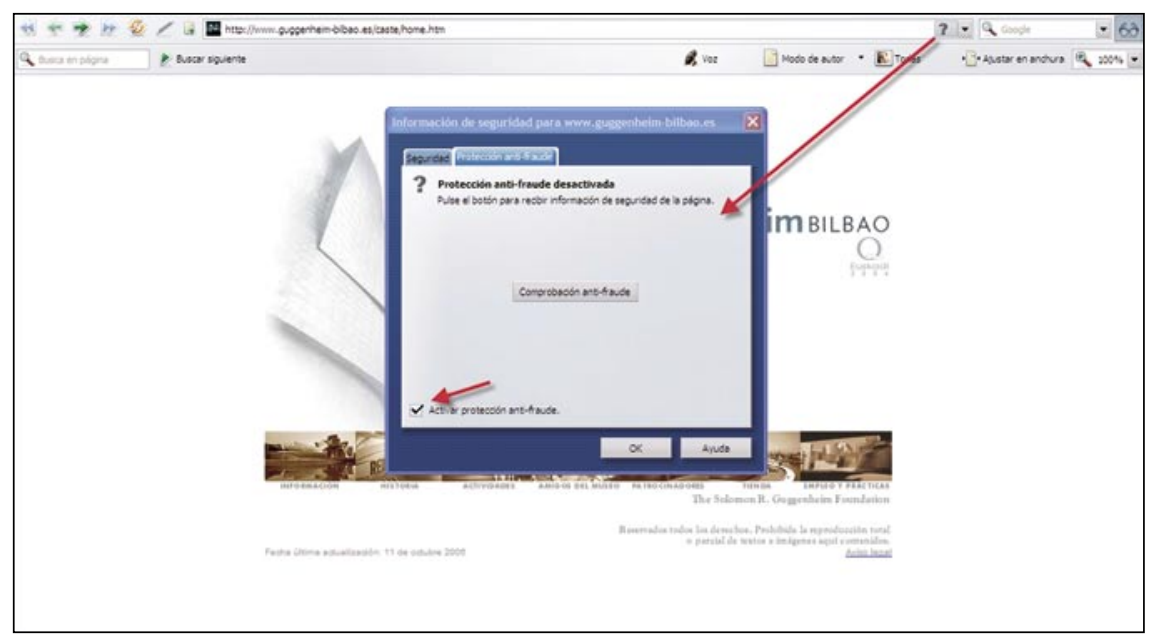

Figura 2. Activación de la protección anti-fraude de Opera

se visita un sitio web. ¿En que se basan para saber si una página es realmente lo que dice ser? Disponen de las características actualizadas de código fuente, metadatos, $\mathrm{xml}$, etc. de las páginas oficiales de diversas instituciones sensibles. Por ejemplo, cuando se visita la Agencia Tributaria, el filtro coteja las características e indicará, en caso necesario, el peligro de suplantación. En sentido contrario, estos navegadores también disponen de listas de sitios fraudulentos. En Opera esta protección es opcional, pues es necesario activar previamente el filtro (figura 2).

Firefox ofrece listados de datos procedentes de Google que se instalan como una extensión de esta función. Además, los tres navegadores añaden otras medidas de seguridad activa relacionadas con la bajada de archivos, aplicaciones Java, controles, Activex, cookies, certificados caducados, etc. Por su parte, Opera también ofrece aplicaciones extra de seguridad, como por ejemplo la posibilidad de bloquear el contenido de un sitio desde el propio ratón o la de no permitir la descarga de ninguna imagen. En conjunto, podemos decir que los tres navegadores han asumido de una forma mucho más eficiente la protección de los usuarios. Cabe destacar, no obstante, que el mérito histórico por esta preocupación corresponde a Firefox.

\section{"Explorer, Firefox y Opera han asumido de una forma mucho más eficiente la protección de los usuarios"}

\section{Navegación}

En opinión de los analistas, probablemente la utilidad más práctica que ha surgido de los navegadores de nueva generación es la gestión por pestañas. Hasta ahora si un usuario de Explorer 6 deseaba abrir un vínculo teniendo ya abierta una página web, indefectiblemente se abría en una nueva ventana. La nueva versión posibilita que lo haga en una nueva pestaña dentro de la misma ventana de trabajo. Por lo tanto aumenta su visibilidad y se reduce el tiempo de acceso.

$\mathrm{Si}$ seguimos con Explorer, cuando el elevado número de pes- tañas impide leer el título de la web, existe la posibilidad de contemplarlas en conjunto en forma de miniatura en una página que genera de forma automática. Todo ello con un simple clic en la pestaña situada en la parte izquierda de la barra. Asimismo, este mismo número de pestañas abiertas puede guardarse conjuntamente en los favoritos.

En este apartado Explorer destaca en comparación con los otros dos navegadores en liza aunque, de todas formas, el uso cada vez más generalizado de favicons (favorite icons, iconos identificativos del sitio) posibilita el reconocimiento rápido en cualquier de los tres navegadores.

Mientras que Explorer y Ope$r a$ ofrecen la barra de pestañas por defecto, en Firefox debe crearse en el menú de herramientas. Tampoco ofrece la opción de guardar la sesión de trabajo con las diversas pestañas abiertas (aunque sí llevar todo el grupo de pestañas a "Marcadores"); una opción que está presente en Explorer y que Opera ofrece por defecto.

Otros perfeccionamientos apreciables radican en el aumento de la superficie del navegador destinada a la visualización de la página y una simplificación en la distribución de las herramientas, más apreciable en el caso de Explorer y menos en $\mathrm{Fi}$ refox.

En cuanto a Opera debemos destacar creaciones que no presentan los demás: añadir más funciones a los clásicos botones de "adelante" y "atrás" e "inicio", así como poder ir a la primera o última página web visitada en la sesión. Todas estos avances, una vez pagado el peaje de su curva de aprendizaje, deparan un gran ahorro de tiempo en la navegación (figura 3 ). 
Integrar la función de buscador corrige una carencia en Explorer, y es una buena continuidad en el caso de Firefox y Opera. Explorer adolece de la posibilidad de intercalar diferentes buscadores en función de la necesidad, pues sólo ofrece cada vez un buscador por defecto. Una cuestión mucho mejor resuelta en las otras dos aplicaciones.

Opera añade una opción exclusiva: facilita la interacción del navegador mediante voz, lo que aporta un enorme valor para algunas discapacidades. Posee funciones programables, activa diversos comandos (tales como abrir página, guardar, atrás, adelante, escoger parte del texto, etc.), aunque se debe disponer de una buena pronunciación en inglés. Esperamos que aparezcan pronto iniciativas que traduzcan esta función a otras lenguas. En el apartado sobre accesibilidad examinaremos otras cuestiones relacionadas.

http://www.opera.com/products/desktop/

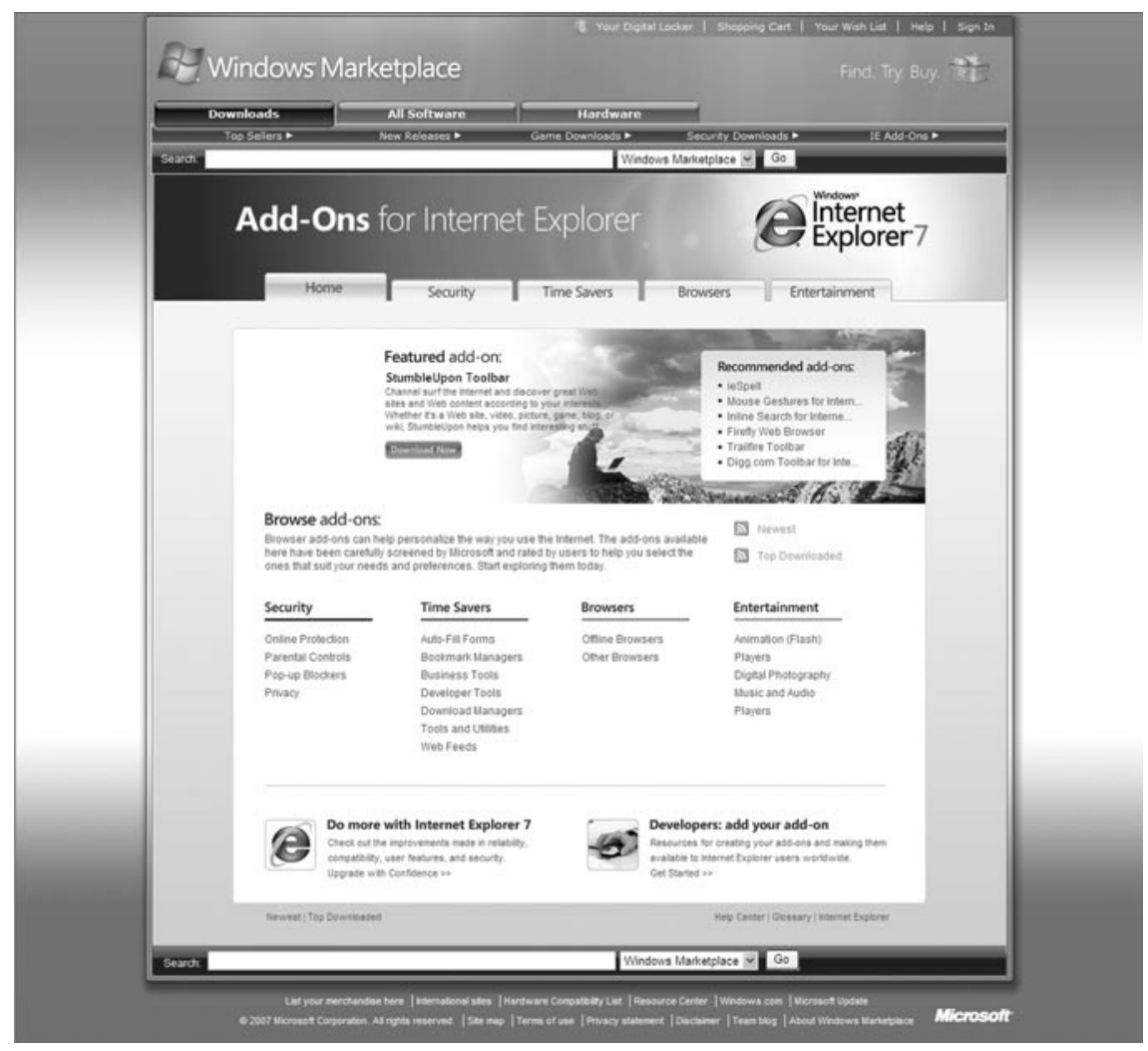

Figura 4. Página principal del sitio web add-ons de Explorer

\section{Personalización}

Los usuarios podían añadir herramientas adicionales a los navegadores anteriores en función de sus necesidades (page rank, traffic rank, buscadores, etc.) siempre $\mathrm{y}$ cuando supieran y buscaran el sitio web correspondiente (Google, Alexa, Yahoo!), seleccionaran su barra de herramientas, la descargaran y la instalaran.

En la nueva generación de navegadores la situación ha cambiado con el concepto de las agregaciones o extensiones, creación original de Firefox (add-ons), que ahora lo incluyen también los otros dos, aunque las opciones varían según el caso. Veámoslas por separado.

Explorer no es el que ofrece el mayor número de extensiones ( $a d d$ ons) pero se diferencia en su presentación y en su oferta mixta tanto gratuita como de pago. El sitio web que facilita su uso e instalación resulta muy práctico e intuitivo, en particular por la clasificación indicadora que ofrece Microsoft a los usuarios en función de cuatro grandes temas: "seguridad, ahorradores de tiempo, navegadores y entretenimiento", sin descontar que debajo de cada ítem añade descripciones más específicas.

La clasificación no acaba aquí: una vez entramos en cualquiera de las categorías mencionadas, cabe la posibilidad de discriminar su listado de productos por diversas variables, tales como precios, categoría más cercana a las necesidades del usuario, por fabricante, por el más reciente y por el más popular.

Firefox es el que dispone de un mayor número de agregaciones. Una de las razones tiene que ver con la propia filosofía para su construcción: el código abierto. Precisamente su libertad de acceso permite a cualquier diseñador construir su propia agregación o extensión y ofrecerla en el sitio de Firefox.

$\mathrm{Al}$ contrario que el resto de navegadores, Mozilla establece dos categorías de complementos. Los ya citados add-ons o agregaciones y las extensions. Según la propia fundación se trata de "agregaciones de pequeño tamaño que añaden nuevas funcionalidades al navegador Firefox". Por lo tanto, la diferencia entre ambas parece radicar en su tamaño y entran en la categoría genérica de add-ons.

En cuanto a Opera, lo más destacado es su forma de presentar las extensiones (que denomina widgets) en su web: grandes iconos sobre fondo blanco que resultan muy agradables visualmente. Es posible seleccionar cualquiera de sus agregaciones sobre la base de una variada clasificación: la del propio editor de la web, las más votadas por los usuarios, las más descargadas, por diferentes categorías, por su autor y por las más actuales.

Aunque los tres navegadores facilitan construir un nuevo complemento o extensión, Opera es el único que ofrece un tutorial para 


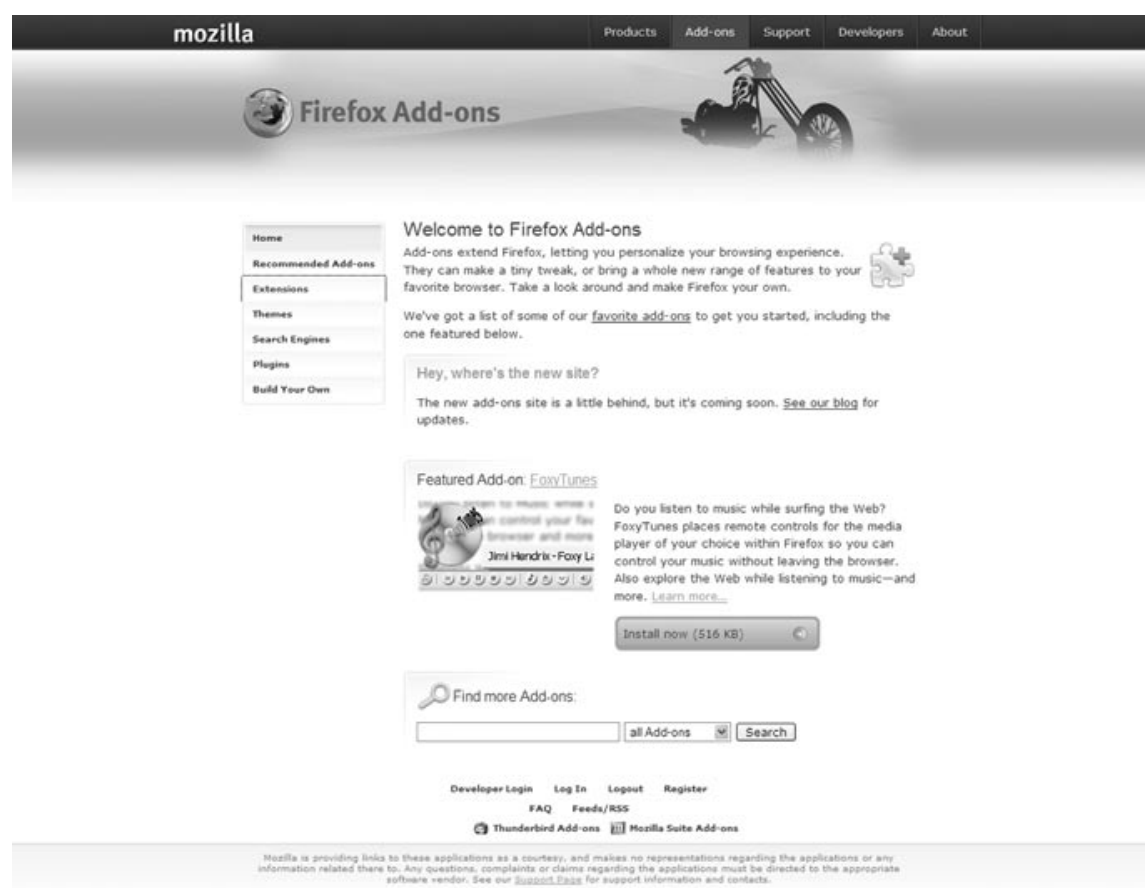

Figura 5. Página principal sitio web add-ons de Firefox

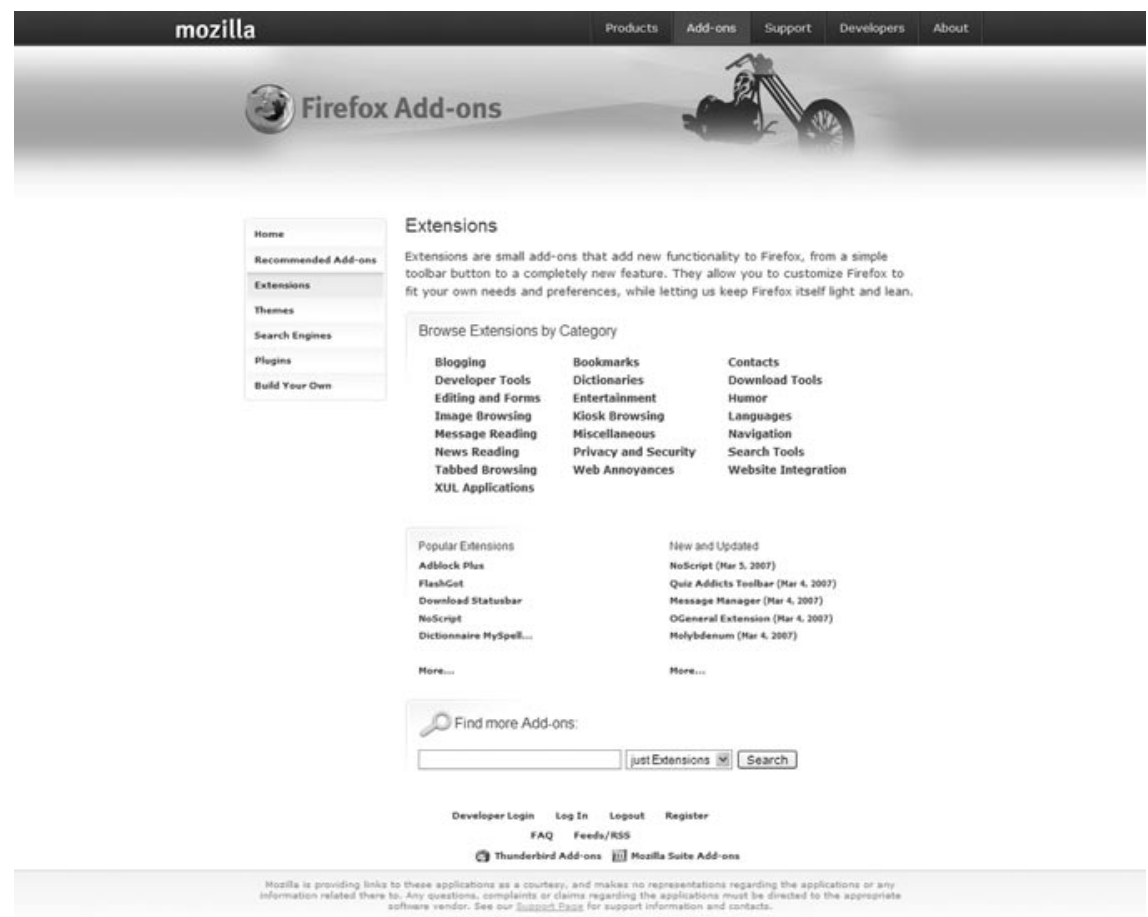

Figura 6. Página web de extensions de Firefox

ello, sin importar el nivel previo y que, tal como ellos mismos prome- ten, permite crear una aplicación en segundos.

\section{Accesibilidad}

Todo sitio web debería tener en cuenta su accesibilidad según las normas del $W 3 C$, como mínimo a nivel A. Precisamente por esa misma razón resulta muy atractivo que sea el propio navegador quien ofrezca diversas herramientas de accesibilidad. Por ejemplo, la aplicación de lupa posibilita aumentar la imagen de la pantalla hasta un $400 \%$ en el caso de Explorer y, un $1000 \%$ en Opera. Esta opción no está contemplada en la actual versión de Firefox.

Pero si hablamos de diversidad de herramientas, Opera destaca claramente por su combinación de utilidades de usabilidad y de accesibilidad. Además del ya citado uso de la voz, en una misma barra de herramientas podemos encontrar otras funciones tales como los diferentes modos de navegación (en la imagen de la figura 8: "Modo de autor"). Es una herramienta que permite usar hasta diez tipos diferentes y complementarios (alto contraste, modo de accesibilidad, analizar estructura, sólo imágenes, etc.) y que cubre en la mayor parte de los casos las necesidades de accesibilidad. También es posible adaptar la imagen al ancho de la pantalla (útil para todos los tamaños), la lupa y quitar la barra de herramientas a fin de aumentar la área de visión.

\section{Conclusiones}

A lo largo del texto hemos ido desgranando algunas particularidades sobre los nuevos navegadores Explorer, Firefox y Opera a partir de la excelente excusa que nos brinda la idea de la web 2.0.

Si nos atenemos a la propia descripción de los fabricantes, los tres han hecho esfuerzo para ser com-

\section{Leer EPI es como asistir a un curso de formación continua pero con el horario acomodado a tus necesidades.}




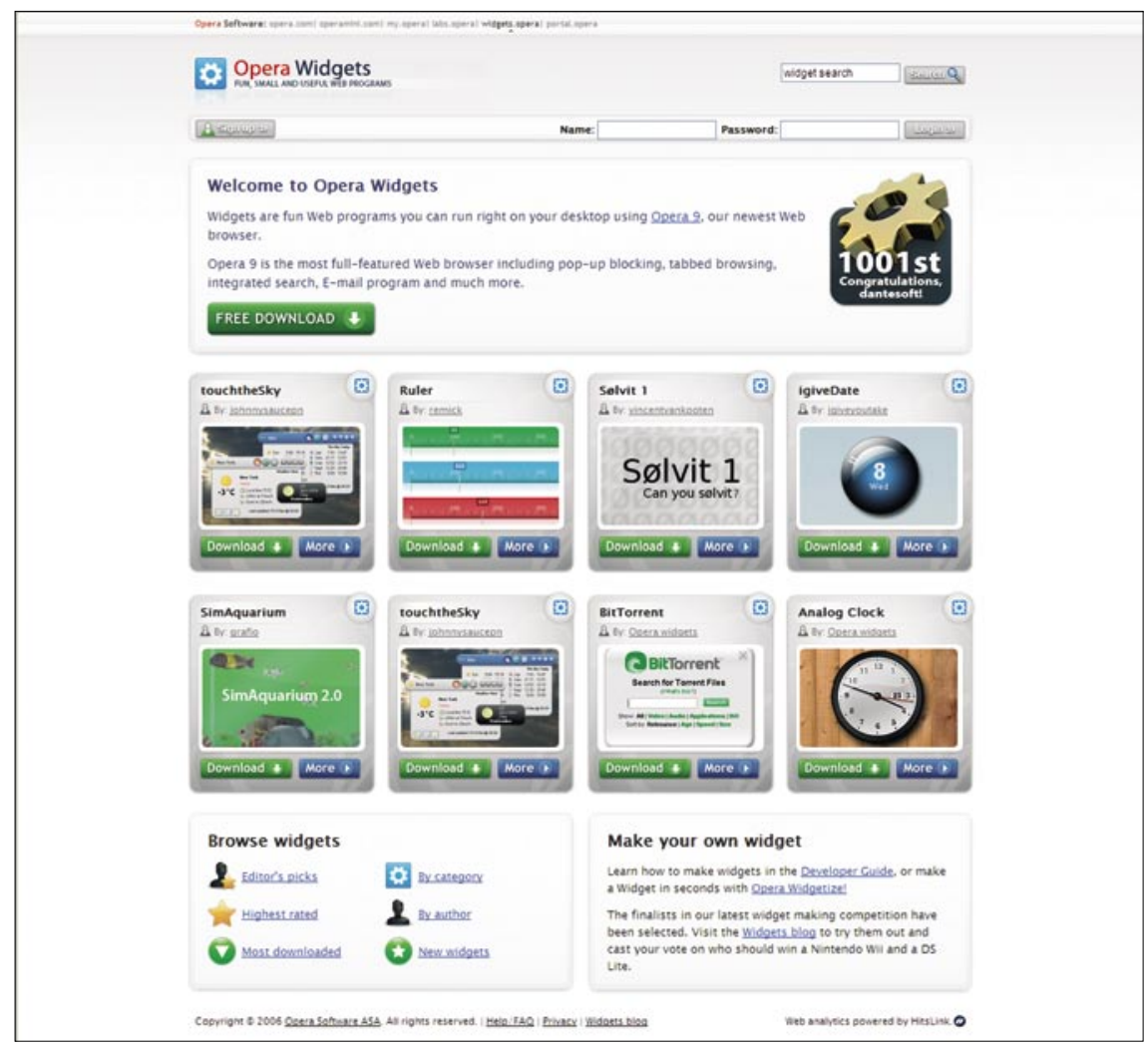

Figura 7. Pagina principal sitio web widgets de Opera

? Q $\mathbf{Q}_{\text {Google }} \longrightarrow 62$

Voz Vor Todo de autor - D. Ajustar en anchura $900 \%$ -

Figura 8. Detalle de los diferentes elementos de accesibilidad situados en la barra de herramientas de Opera

\section{"En nuestra opinión, hoy por hoy, el mejor navegador es Opera"}

patibles con los estándares y las tecnologías de la web actual. Aunque no todos hayan conseguido el mismo grado de eficiencia, hay que valorar este aspecto en su agenda de preocupaciones. En este sentido la mayor parte de los analistas parece estar de acuerdo en que la mayor garantía de compatibilidad la ofrecen Opera y Firefox (por este orden), al menos en lo que se refiere a xhmtl y css

Los tres han adoptado algunas aplicaciones de usabilidad y accesibilidad que facilitan enormemente la gestión de la navegación (pestañas y lupa), sin olvidar las grandes posibilidades de personalización que ofrecen los complementos (add-ons, extensions y widgets). Igualmente, los tres han añadido importantes funciones de seguridad.

Pero si tenemos que combinar facilidad de uso, accesibilidad, facilida-

\section{MAYO 2007} BARCELONA WWW.COBDC.ORG/USID/2007/CAST.HTML

\section{Simplicity is the wltimate

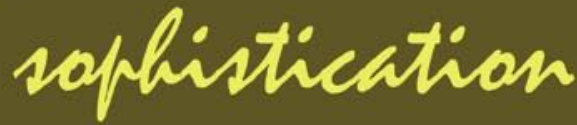

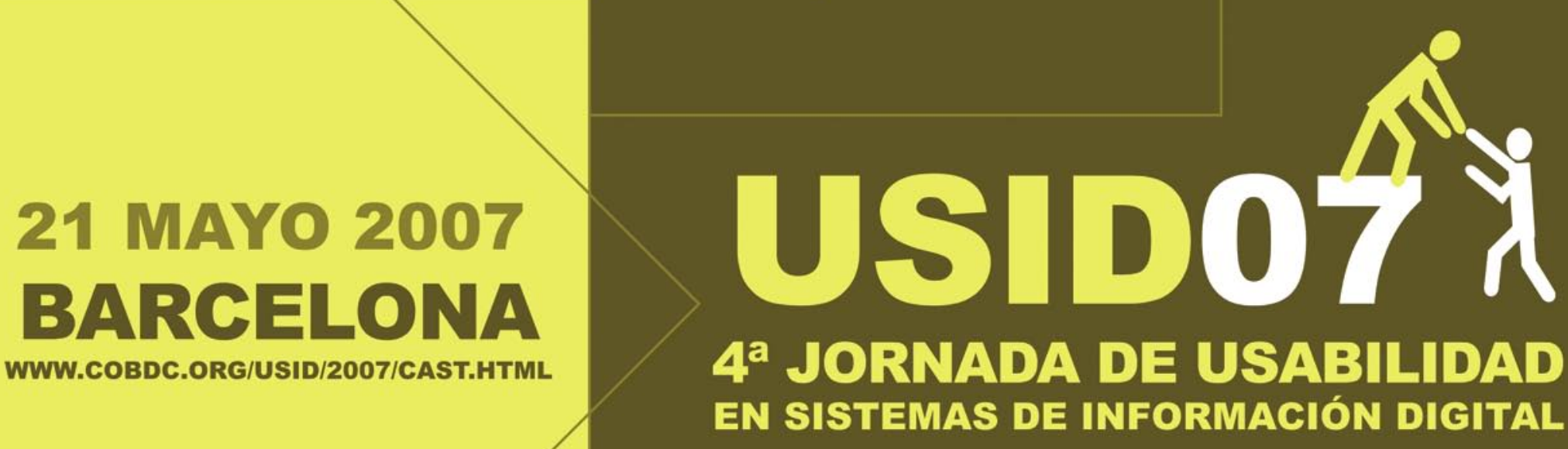


des de navegación y presentación de la información, destaca sin duda Opera sobre los otros dos. Su función de activación por voz, la posibilidad de parar la descarga de una página desde el ratón, la opción de adecuar el modo de navegación a las necesidades de cada usuario de forma permanente o la claridad visual de los iconos de la barra de herramientas lo sitúan en una posición muy ventajosa sobre los otros dos. Dicho de otro modo y para que quede tan claro como nos es posible: en nuestra opinión, hoy por hoy, el mejor navegador de la Web es Opera. Por tanto, la buena noticia es que ahora la comunidad de desarrolladores de Firefox tiene un nuevo aliciente: seguro que hasta ahora les resultaba decepcionantemente fácil derrotar a Explorer.

\section{Fuentes}

Browsers go head-to-head. BBC. Consultado en: 21-02-07.

http://news.bbc.co.uk/2/hi/technology/6086798.stm

"Comparison of web browsers". Wikipedia. Consultado en: 02-3-07.

http://en.wikipedia.org/wiki/Comparison_of_ web_browsers\#_note-0

Firefox browser for web 2.0 age. BBC. Consultado en: 21-02-07.

http://news.bbc.co.uk/2/hi/technology/6078016.stm

"Freeware browsers". Comcast. Consultado en: 23-02-07.

http://mywebpages.comcast.net/SupportCD/ FreewareBrowsers.html

"Opera (navegador)". Wikipedia. Consultado en: 23-02-07.

http://es.wikipedia.org/wiki/Opera (navegador)\#Caracter.C3.ADsticas_.28v_8.5x.29

"The state of web 2.0". Dion Hinchcliffe. Consultado en: 22-02-07.

http://web2.wsj2.com/the_state_of_web_20.htm

“Web 2.0”. Wikipedia. Consultado en: 22-02-07. http://en.wikipedia.org/wiki/Web_2

"Web browser standards support". Web Devout Consultado en: 22-02-07.

http://www.webdevout.net/browser-support

Creamos espacios virtuales avanzados para hacer realidad la preservación y la gestión del conocimiento

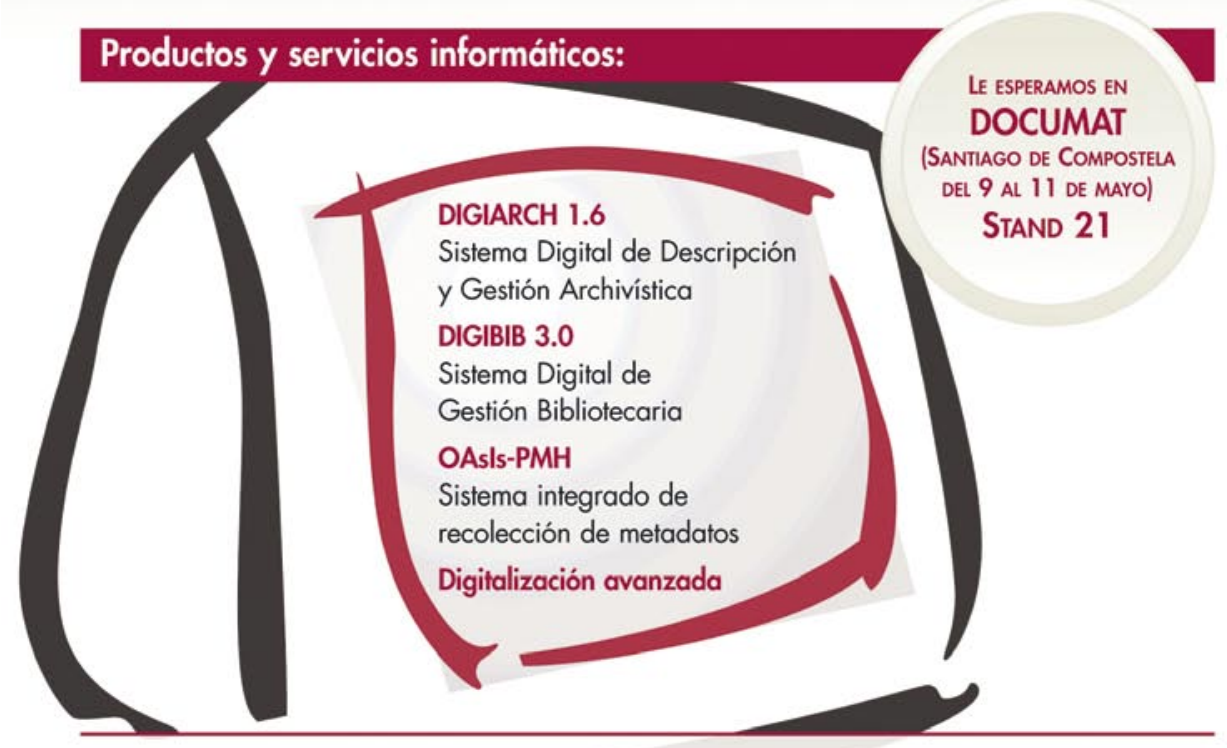

- Tecnologías abiertas para la creación, gestión, búsqueda y recolección de datos y metadatos en XML (MARC21, EAD, METS)

- Recolección e intercambio de datos en la Web para Entidades e Instituciones de Memoria en OAI-PMH y Dublin Core

- Repositorios Institucionales para Preservación Digital a largo plazo mediante OAIS y PREMIS

\section{Visítenos también en} N/Na

\section{0} 10

"Which new browser is best: Firefox 2, Internet Explorer 7, or Opera 9?”. En: Extreme Tech. Consultado en: 23-02-07.

http://www.extremetech.com/article2/ 0,1697,1990850,00.asp

Ricard Monistrol, periodista y consultor independiente. rmonistrol@gmail.com www.ricardmonistrol.cat

\section{Lluís Codina, Universidad Pompeu} Fabra.

lluis.codina@upd.edu www.lluiscodina.com

\section{Suscripciones}

Renovar (o comenzar) la suscripción a "El profesional de la información" es ágil y sencillo. Usted puede gestionar online su suscripción conectándose a esta web: http://www. elprofesionaldelainformacion.com/suscripciones.htm/

Si lo desea puede comunicar con nosotros dirigiéndose a: suscripciones@elprofesionaldelainformacion.com 